\title{
Anticancer Effect of Ginseng Leaves Crude Polysaccharides on Human Hepatoma Cell SMMC-7721
}

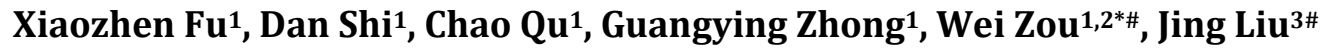 \\ ${ }^{1}$ College of Life Sciences, Liaoning Normal University, Dalian, China \\ ${ }^{2}$ Liaoning Key Laboratories of Biotechnology and Molecular Drug Research and Development, Dalian, China \\ ${ }^{3}$ The First Affiliated Hospital, Dalian Medical University, Dalian, China \\ Email: weizou60@126.com
}

Received 21 April 2014; revised 22 May 2014; accepted 2 June 2014

Copyright (C) 2014 by authors and Scientific Research Publishing Inc.

This work is licensed under the Creative Commons Attribution International License (CC BY).

http://creativecommons.org/licenses/by/4.0/

(c) (i) Open Access

\section{Abstract}

Background: Polysaccharides extracted from the medicinal herbs are known to possess antitumor effects. Although there is a significant number of evidences implicated on the beneficial effects of the ginseng leaves ginsenoside with diverse associated mechanisms, reports on the anticancer by the ginseng leaves crude polysaccharides (GLCP) are not sufficient. Experiments were carried out to unravel the anticancer effects of GLCP. Methods: Cells were treated with GLCP $(0.5-2 \mathrm{mg} / \mathrm{ml})$ for $48 \mathrm{~h}$. MTT method was used to detect the cell viability. Western blot and flow cytometry were used to detect apoptotic rate. Western blot and acridine orange staining were used to detect the cell autophagy. Results: Compared with the normal human liver cell (Chang liver), GLCP (1.5 - 2 $\mathrm{mg} / \mathrm{ml}$ ) significantly reduction cell viability, promote apoptosis-related proteins expression, promote cell apoptosis and autophagy in SMMC-7721 cells. But caveolin-1 gene silencing could inhibit the anticancer effect of GLCP. Conclusions: These data suggest that GLCP promote autophagy and apoptosis in human hepatoma cell SMMC-7721. We speculate that its mechanism may be associated with the caveolin-1 which is an essential structural molecule of caveolae. Although the effect of GLCP inhibited of liver cancer is not very strong, we are more interested in the GLCP which plays a tumor suppressor role in health care.

\section{Keywords}

Anticancer, Ginseng Leaves Crude Polysaccharides (GLCP), Caveolin-1, Autophagy, Apoptosis

\footnotetext{
${ }^{*}$ Corresponding author.

${ }^{\#}$ Jing Liu and Wei Zou contributed equally to this work.
} 


\section{Introduction}

Hepatoma is currently the sixth most common type of cancer and the third leading cause of cancer-related death in the world [1]. In China, more than 401,000 new patients are diagnosed with hepatoma and more than 371,000 patients are killed by the terrible disease annually [2]. Although recent advances in chemotherapeutic agents in hepatoma have been achieved, treatment options are still limited and are associated with significant morbidity and mortality.

Ginseng is a kind of traditional Chinese medicine, ginseng polysaccharides which because of its anti-tumor, anti-oxidation, hypoglycemic, and many other activities was widespread concern in recent years [3] [4]. The ginseng root requires 6 - 7 years to harvest, while ginseng leaves can be harvested annually; recent studies have shown that there was the same active ingredient in the ginseng and ginseng leaves, such as fatty acids, alkaloids, polysaccharides, sesquiterpenes, ginsenosides and so on [5] [6]. But the ginseng leaves activities especially the anticancer activity have not been reported.

In the present study, we have investigated the anticancer effects of ginseng leaves crude polysaccharides (GLCP) in human hepatoma cell SMMC-7721. Herein, we show that GLCP promote cell apoptosis and autophagy in SMMC-7721 cells. Furthermore, we demonstrated that caveolin-1 gene silencing inhibits the anticancer effect of GLCP. So its mechanism may be associated with the caveolin-1 which is an essential structural molecule of caveolae.

\section{Materials and Methods}

\subsection{Materials}

RPMI-1640 medium, Opti-MEM ${ }^{\circledR}$ reduced serum media and newborn bovine serum (NBCs) were purchased from Gibco Laboratories (Grand Island, NE, USA). Penicillin and streptomycin were purchased from Invitrogen (Carlsbad, CA, USA). The primary antibodies were purchased from Cell Signaling Technology (Boston, MA, USA).

\subsection{Cell Lines and Culture Condition}

The human hepatoma cell (SMMC-7721) and normal human liver cell (Chang liver) were obtained from the Cell Bank of the Institute of Biochemistry and Cell Biology, China Academy of Sciences (Shanghai, China). Cav-1 knockdown hepatoma SMMC-7721 cells using RNA interference technology to build early in our laboratory. The cells were grown in RPMI-1640 medium containing 15\% newborn bovine serum (NBCs), penicillin $(100 \mu \mathrm{g} / \mathrm{ml})$, and streptomycin $(100 \mu \mathrm{g} / \mathrm{ml})$. Cultures were maintained at $37^{\circ} \mathrm{C}$ in a humidified $5 \% \mathrm{CO}_{2}$ atmosphere. Ginseng leaves crude polysaccharides (GLCP) was dissolved in the complete medium and adjusted to the indicated final concentrations with culture medium before use.

\subsection{Cell Viability Measurement Assay}

The effect of GLCP on the growth of human hepatoma cell SMMC-7721 was evaluated using $5 \times 10^{3}$ cells seeded onto 96-well plates, which were treated with GLCP after $24 \mathrm{~h}$ at the time of cell plated. To evaluate the effect of GLCP at concentrations $0.5,1,1.5$, and $2 \mathrm{mg} / \mathrm{ml}$, cells were maintained in media with various concentrations of GLCP for $48 \mathrm{~h}$ and cell numbers were determined by 3-[4,5-dimethylthiazol-2-yl]-2,5-diphenyltetrazolium bromide (MTT) assay, and absorbance was read at $570 \mathrm{~nm}$.

\subsection{Western Blot Analysis}

Cells were plated in 6-well plates and were treated with GLCP for $48 \mathrm{~h}$. After treatment, the cells were harvested and resuspended in ice-cold cell lysis buffer, and the homogenate was centrifuged at $10,000 \mathrm{~g}$ for 15 min at $4^{\circ} \mathrm{C}$. The total supernatant protein concentration was measured using a bicinchoninic acid (BCA) protein assay kit (Kangchen Biotech, Shanghai, China). An equal volume of sodium dodecyl sulfate (SDS) gel loading buffer [100 mM Tris-HCl (pH 6.8), 200 mM dithiothreitol (DTT), 4\% SDS, 0.1\% bromophenol blue and 20\% glycerol] was added to samples, which were subsequently boiled for $7 \mathrm{~min}$. The total proteins from each sample were separated by 15\% sodium dodecylsulfate-polyacrylamide gel electrophoresis (SDS-PAGE) and were transferred to a polyvinylidene difluoride (PVDF) membrane. The membrane was blocked with 5\% fat-free dry milk in PBS-T 
for $1 \mathrm{~h}$ at room temperature followed by incubation with primary antibodies to caveolin-1 (1:1000), LC3B (1:1000), Bcl-2 (1:1000), P53 (1:1000) and $\beta$-actin (1:2000) overnight at $4^{\circ} \mathrm{C}$. The next day, the membranes were washed and subsequently incubated with HRP-conjugated secondary antibodies for $2 \mathrm{~h}$ at room temperature. Following three washes with PBS-T, the membranes were developed using an enhanced chemiluminescence (ECL) kit and exposed to X-ray films. Gel-Pro analyzer 4 software was used to quantify the protein expression levels.

\subsection{Acridine Orange Staining}

Cells were plated in 24-well plates and were treated with GLCP for 48 h. After washed twice with phosphate buffered saline (PBS), the cells were fixed with 95\% ethanol for $10 \mathrm{~min}$. Acridine orange was added at a final concentration of $0.1 \mathrm{mg} / \mathrm{ml}$ for $25 \mathrm{~min}$ at $4^{\circ} \mathrm{C}$, after washed twice with PBS, added $0.1 \mathrm{~mol} \mathrm{CaCl}_{2}$ in the cells for colour separation. Finally, cells were washed with phosphate buffered saline. Pictures were obtained with a fluorescence microscope.

\subsection{Flow Cytometry}

Cell apoptosis was quantified by flow cytometry using PI and Annexin V double staining, and it was performed as previously described using FACScalibur (Becton Dickinson, Franklin Lakes, NJ).

\subsection{Statistical Analysis}

All of the experiments were performed at least three times. The values were expressed as the means \pm S.E.M. Statistical analysis was performed between groups by ANOVA using SPSS 16.0 software (SPSS Inc, Chicago, IL, USA). A probability value of $p<0.05$ was considered significant.

\section{Results}

\subsection{Ginseng Leaves Crude Polysaccharides Promote Apoptosis in Human Hepatoma Cell SMMC-7721}

MTT analysis showed that compared with the control group, the relative viability of SMMC-7721 cell decreased significantly after adding different concentrations $(0.5,1,1.5$, and $2 \mathrm{mg} / \mathrm{ml})$ of GLCP, at the same time, GLCP activity of normal liver cells in some role in promoting (Figure 1(a)). Using flow cytometry detection after adding the $1.5 \mathrm{mg} / \mathrm{ml}$ polysaccharide on apoptosis in SMMC-7721 cells, the results showed that, adding polysaccharide treatment significantly increased apoptosis rate (Figure 1(b)). Western blot to detect the apoptosis associated protein expression changes after adding the GLCP in human hepatoma cell SMMC-7721. We can see from the quantitative, GLCP inhibits the expression of BCL-2 proteins in human hepatoma cell SMMC-7721 and promotes the expression of NF- $\kappa$ b (Figure 1(c)).

\subsection{GLCP Promote Human Hepatoma Cell SMMC-7721 Autophagy}

To determine if GLCP induced autophagy in SMMC-7721 cells, acridine orange staining for autolysosome was performed. Results showed that the GLCP at the concentration of $1.5 \mathrm{mg} / \mathrm{ml}$ drastically increased the formation of autolysosome in SMMC-7721 cells (Figure 2(a)).Western blot showed the marker protein levels of autophagy, compared with control group the LC3-II/LC3- I level was increased significantly after adding $1.5 \mathrm{mg} / \mathrm{ml}$ GLCP (Figure 2(b)).

\subsection{Caveolin-1 Gene Silencing Inhibits the Anticancer Effect of GLCP}

MTT method and flow cytometry were used to detect the anticancer effect of GLCP in caveolin-1 gene silencing SMMC-7721 cells. Compared with the SMMC-7721 cells, the relative viability of caveolin-1 gene silencing SMMC-7721 cells basically unchanged after adding different concentrations (0.5, 1, 1.5, and 2 mg/ml) of GLCP (Figure 3(a)). Using flow cytometry detection after adding the $1.5 \mathrm{mg} / \mathrm{ml}$ polysaccharide on apoptosis in SMMC-7721 cells and the caveolin-1 gene silencing SMMC-7721 cells, the results showed that, adding polysaccharide treatment the apoptosis rate of the caveolin-1 gene silencing SMMC-7721 cells was unchanged 


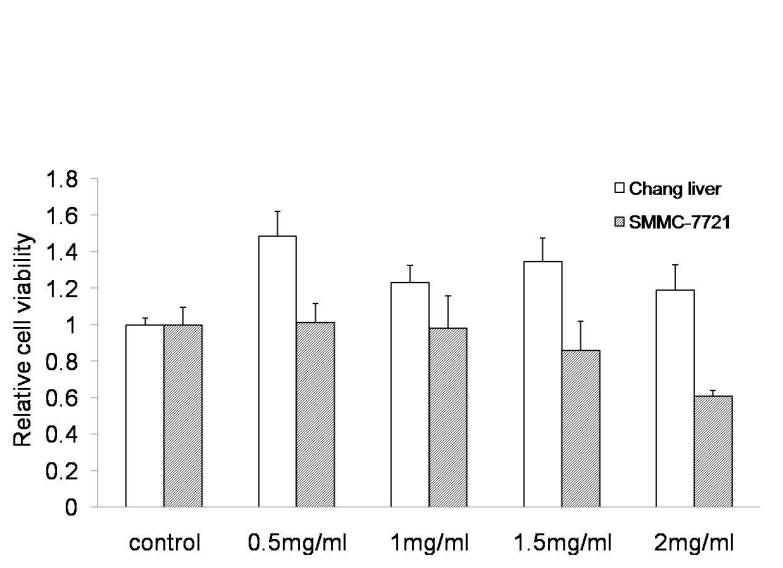

(a)

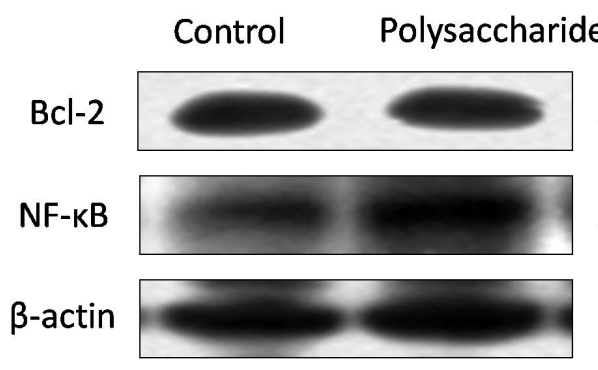

28KDa

50KDa

43KDa

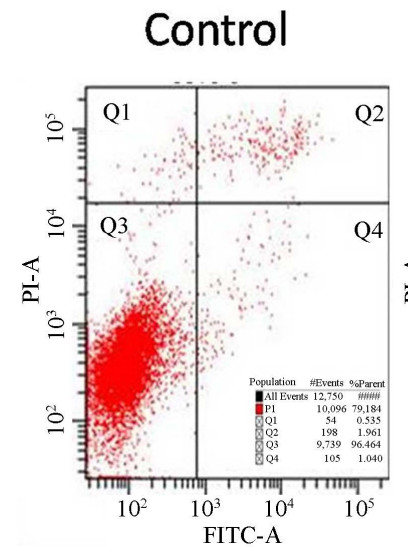

\section{Polysaccharide}

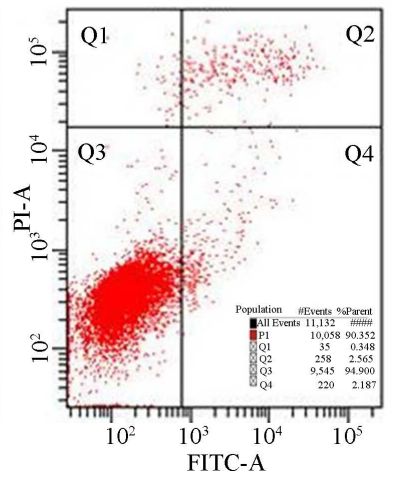

(b)

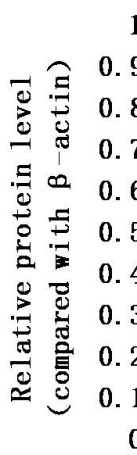

(c)

Figure 1. Compared with the normal human liver cell (Chang liver), GLCP promote cell apoptosis in human hepatoma cell SMMC-7721. (a) The cells were treated with $0.5-2 \mathrm{mg} / \mathrm{ml}$ GLCP for $48 \mathrm{~h}$. Cell viability was measured by the MTT assay as described in methods. (b) The SMMC-7721 cell was treated with $1.5 \mathrm{mg} / \mathrm{ml}$ GLCP for $48 \mathrm{~h}$. The cell apoptosis level was examined by flow cytometry. (c) The SMMC-7721 cell was treated with $1.5 \mathrm{mg} / \mathrm{ml} \mathrm{GLCP}$ for $48 \mathrm{~h}$. Western blot was used to examine the relative protein expression of $\mathrm{Bcl}-2$ and $\mathrm{NF}-\kappa \mathrm{B}$, the protein level was compaerd with the $\beta$-actin. The data represent the mean of three independent experiments $\pm \mathrm{SD} .{ }^{*} p<0.05 ;{ }^{* *} p<0.01$ versus control.

(Figure 3(b)). Western blot to detect the apoptosis associated protein expression changes after adding the GLCP in human hepatoma cell SMMC-7721 and the caveolin-1 gene silencing SMMC-7721 cells. The results showed that, caveolin-1 gene silencing inhibits the anticancer effect of GLCP.

\section{Discussion}

Hepatocellular carcinoma (HCC) is widespread primary malignant tumor of the liver. It is the third and ninth most common cause of cancer associated deaths in men and women, respectively [7]. Current anti-tumor treatments, such as radiotherapy, chemotherapy, immunotherapy, and suicide gene therapy, act on tumor by inducing tumor cell apoptosis [8]. Chemotherapy is the most common treatment option for hepatoma [9]. However, its effectiveness is often compromised because of inherent or acquired drug resistance, with major impediment being frequent appearance of multi-drug resistant cancer cells during drug treatment [10]. So looking for non-toxic or low toxic anti-cancer drugs from natural products become a new hotspot in recent years [11] [12].

A variety of active polysaccharide extracted from plants can influence the humoral immunity, innate and adaptive immunity. Now, the lentinan and polysaccharides of ganoderma lucidum, bidentata, polyporus, huangqi and etc are commonly used in clinical [13]. However, the activity of plant polysaccharides not only in strengthening the immune system as concerned, the same has anti-cancer effect [14] [15]. Use of plant polysaccharides as anticancer health food has long been applied in our country, but due to its mechanism of action is not clear, this treatment still cannot be received by the international community. 

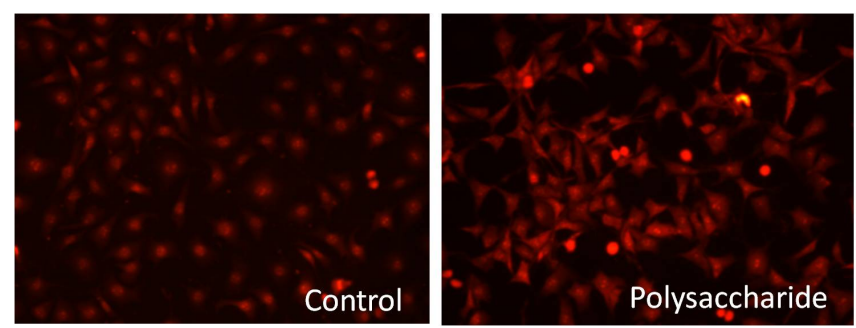

(a)

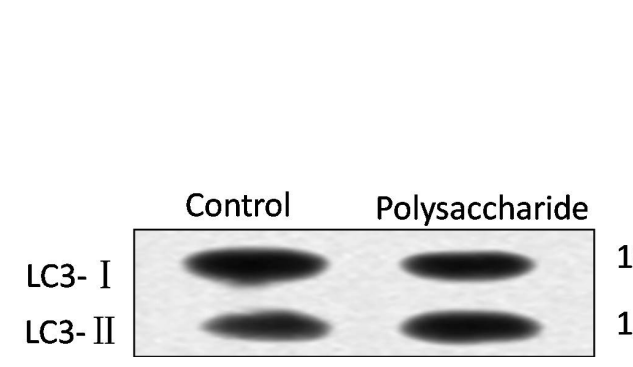

$18 \mathrm{KDa}$

16KDa

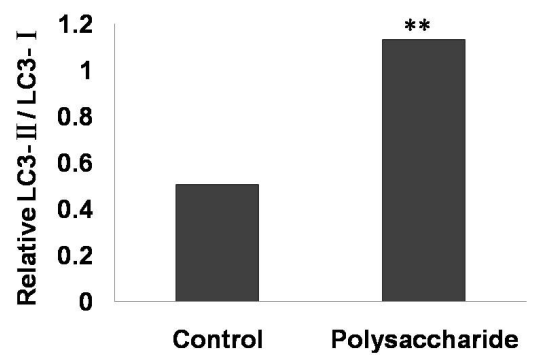

(b)

Figure 2. Induction of autophagy in human hepatoma cell SMMC-7721 after treatment with GLCP. SMMC-7721 cells were treated with $1.5 \mathrm{mg} / \mathrm{ml}$ GLCP for $48 \mathrm{~h}$. (a) The accumulation of acidic vesicular organelles (AVOs), which emitted bright red fluorescence, was visualized by acridine orange staining; (b) Western blot was used to examine the protein expression of LC3, using LC3-II/ LC3-I to represent the level of the autophagy.

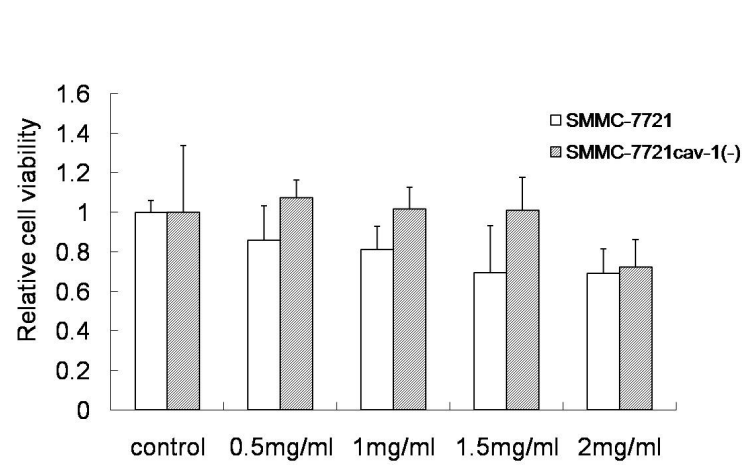

(a)

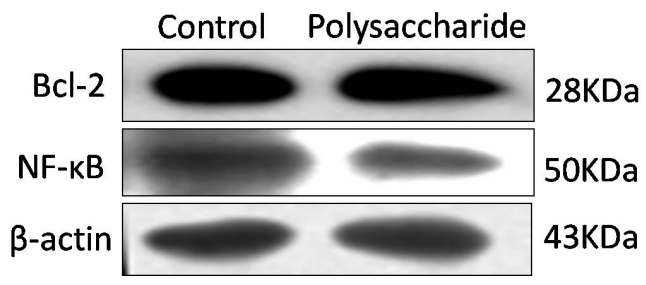

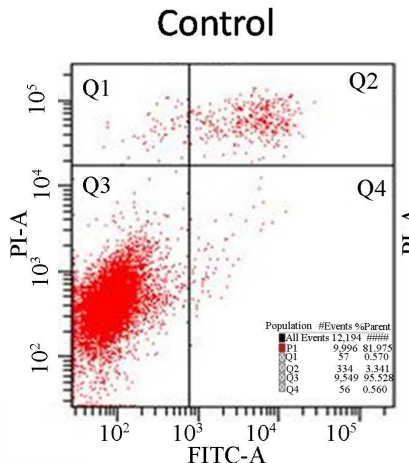

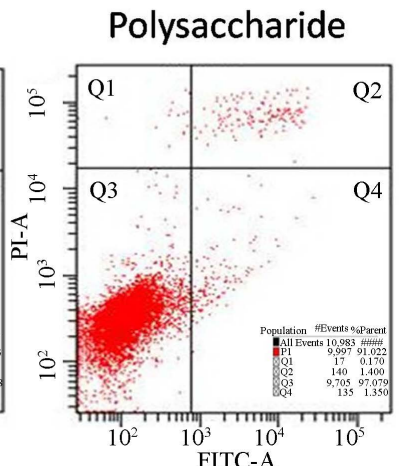

(b)

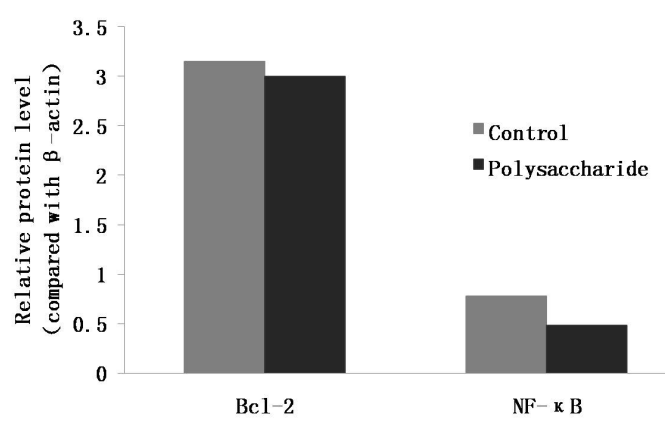

(c)

Figure 3. Compared with human hepatoma cell SMMC-7721, caveolin-1 gene silent resisted GLCP promote the cell apoptosis. (a) The cells were treated with $0.5-2 \mathrm{mg} / \mathrm{ml} \mathrm{GLCP}$ for $48 \mathrm{~h}$. Cell viability was measured by the MTT assay as described in methods. (b) The SMMC-7721 $1^{\text {cav-1(-) }}$ cell was treated with $1.5 \mathrm{mg} / \mathrm{ml} \mathrm{GLCP}$ for $48 \mathrm{~h}$. The cell apoptosis level was examined by flow cytometry. (c) The SMMC-7721 ${ }^{\text {cav-1(-) }}$ cell was treated with $1.5 \mathrm{mg} / \mathrm{ml}$ GLCP for 48 h. Western blot was used to examine the relative protein expression of Bcl-2 and NF- $\kappa \mathrm{B}$, the protein level was compaerd with the $\beta$-actin. The data represent the mean of three independent experiments \pm SD. ${ }^{*} p<0.05 ;{ }^{* *} p<0.01$ versus control. 
Caveolin-1 is a kind of 21 - $24 \mathrm{KD}$ membrane protein, and it is an essential structural molecule of caveolae [16]. Its function are quite diverse, ranging from cellular cholesterol transport, cell membrane composing, cellular signal transduction, cell cycle regulation and control, cell transformation to tumorigenesis [17] [18]. Previously studies in our lab have demonstrated that caveolin-1 gene silencing activates estrogen receptor alpha expression and leads to 17 beta-estradiol-stimulated mammary tumorigenesis [19]. Recent studies have showed that caveolin-1 has close relation with breast epithelial cell transformation and breast tumor formation [20]. Downregulation of caveolin-1 could increase cell apoptosis in vitro [21] [22]. In this paper, caveolin-1 has been shown to participate in the mechanism of GLCP-induced hepatoma cell apoptosis.

In this study we used the traditional extracted method of oriental medicines to obtain the ginseng leaves crude polysaccharides (GLCP) [23]. Ginseng leaves (50 g) was extracted with $1 \mathrm{~L}$ of boiling water for $4 \mathrm{~h}$, after filtered it was precipitated with ethanol and deproteinized by the Sevag method and then lyophilized, producing an average yield of $28.53 \%$. The powdered extract (GLCP) was dissolved in complete medium and then filtered through a $0.22 \mu \mathrm{m}$ syringe filter. Then observe its biological effect on normal liver cells and liver cancer cells.

Our experiments show that GLCP promote apoptosis and autophagy in human hepatoma cell SMMC-7721, at the same time it had no effect on the activity of normal liver cell Chang liver. And its mechanism may be associated with the caveolin-1, because when caveolin-1 gene silencing the anticancer effect of GLCP had been disappeared. Although the results show that the effect of GLCP inhibited of liver cancer is not very strong, here we study primary focus the mechanism of the anticancer effect, and to provide a dietary health reference. In the future studies, we will separate the active ingredients from the GLCP, to looking for the non-toxic or low toxic natural anti-cancer drugs.

\section{Conclusion}

The result shows that GLCP promote autophagy and apoptosis in human hepatoma cell SMMC-7721, and the mechanism may be associated with the caveolin-1 which is an essential structural molecule of caveolae.

\section{Acknowledgements}

This work was supported by a grant from the National Natural Science Foundation of China (No. 30970353), the National Natural Science Foundation of China (No.30570225) and the National Science and Technology Cooperation Project of Ministry of Science and Technology (2010DFR30850).

\section{References}

[1] Gu, W., Fang, F.F., Li, B., et al. (2012) Characterization and Resistance Mechanisms of a 5-Fluorouracil-Resistant Hepatocellular Carcinoma Cell Line. Asian Pacific Journal of Cancer Prevention, 13, 4807-4814. http://dx.doi.org/10.7314/APJCP.2012.13.9.4807

[2] Ferlay, J., Shin, H.R., Bray, F., et al. (2010) Estimates of Worldwide Burden of Cancer in 2008: GLOBOCAN 2008. International Journal of Cancer, 127, 2893-2917.

[3] Kim, J. (2008) Protective Effects of Asian Dietary Items on Cancers-Soy and Ginseng. Asian Pacific Journal of Cancer Prevention, 9, 543-548.

[4] Wee, J.J., Mee Park, K. and Chung, A.S. (2010) Biological Activities of Ginseng and Its Application to Human Health. Source Herbal Medicine: Biomolecular and Clinical Aspects. 2nd Edition. CRC Press, Boca Raton; 2011. Chapter 8.

[5] Yang, S.O., Lee, S.W., Kim, Y.O., et al. (2013) HPLC-Based Metabolic Profiling and Quality Control of Leaves of Different Panax Species. Journal of Ginseng Research, 37, 248-253.

[6] Choi, S.Y., Cho, C.W., Lee, Y., et al. (2012) Comparison of Ginsenoside and Phenolic Ingredient Contents in Hydroponically-Cultivated Ginseng Leaves, Fruits, and Roots. Journal of Ginseng Research, 36, 425-429. http://dx.doi.org/10.5142/jgr.2012.36.4.425

[7] Semela, D. and Heim, M. (2011) Hepatocellular Carcinoma. Therapeutische Umschau, 68, 213-217. http://dx.doi.org/10.1024/0040-5930/a000153

[8] Fulda, S. and Debatin, K.M. (2006) Extrinsic versus Intrinsic Apoptosis Pathways in Anticancer Chemotherapy. Oncogene, 25, 4798-4811. http://dx.doi.org/10.1038/sj.onc.1209608

[9] Wong, R. and Frenette, C. (2011) Updates in the Management of Hepatocellular Carcinoma. Journal of Gastroenterology and Hepatology (N Y), 7, 16-24. 
[10] Mellor, H.R. and Callaghan, R. (2008) Resistance to Chemotherapy in Cancer: A Complex and Integrated Cellular Response. Pharmacology, 81, 275-300. http://dx.doi.org/10.1159/000115967

[11] Boh, B. (2013) Ganoderma Lucidum: A Potential for Biotechnological Production of Anti-Cancer and Immunomodulatory Drugs. Recent Patents on Anti-Cancer Drug Discovery, 8, 255-287. http://dx.doi.org/10.2174/1574891X113089990036

[12] Zong, A., Cao, H. and Wang, F. (2012) Anticancer Polysaccharides from Natural Resources: A Review of Recent Research. Carbohydrate Polymers, 90, 1395-410.

[13] He, X.J., Niu, X.Y., Li, J., et al. (2012) Immunomodulatory Activities of Five Clinically Used Chinese Herbal Polysaccharides. Journal of Experimental and Integrative Medicine, 2, 15-27.

[14] Liu, L.N., Guo, Z.W., Zhang, Y., et al. (2012) Polysaccharide Extracted from Rheum Tanguticum Prevents Irradiation-Induced Immune Damage in Mice. Asian Pacific Journal of Cancer Prevention, 13, 1401-145. http://dx.doi.org/10.7314/APJCP.2012.13.4.1401

[15] Song, K.S., Li, G., Kim, J.S., et al. (2011) Protein-Bound Polysaccharide from Phellinus Linteus Inhibits Tumor Growth, Invasion, and Angiogenesis and Alters Wnt/ $\beta$-Catenin in SW480 Human Colon Cancer Cells. BMC Cancer, 11, 307. http://dx.doi.org/10.1186/1471-2407-11-307

[16] Lajoie, P. and Nabi, I.R. (2012) Lipid Rafts, Caveolae, and Their Endocytosis. International Review of Cell and Molecular Biology, 282, 135-163. http://dx.doi.org/10.1016/S1937-6448(10)82003-9

[17] Boscher, C. and Nabi, I.R. (2012) Caveolin-1: Role in Cell Signaling. Advances in Experimental Medicine and Biology, 729, 29-50. http://dx.doi.org/10.1007/978-1-4614-1222-9_3

[18] Fang, K., Fu, W., Beardsley, A.R., et al. (2007) Overexpression of Caveolin-1 Inhibits Endothelial Cell Proliferation by Arresting the Cell Cycle at G0/G1 Phase. Cell Cycle, 6, 199-204.

[19] Zhang, X., Shen, P., Coleman, M., et al. (2005) Caveolin-1 Gene Silencing Activates Estrogen Receptor Alpha Expression and Leads to 17 Beta-Estradiol-Stimulated Mammary Tumorigenesis. Anticancer Research, 1, 369-375.

[20] Syeed, N., Husain, S.A., Abdullah, S., et al. (2010) Caveolin-1 Promotes Mammary Tumorigenesis: Mutational Profile of the Kashmiri Population. Asian Pacific journal of Cancer Prevention, 11, 689-696.

[21] Zhao, X.N., Ma, C., Cai, X.L., Lei, D.P., Liu, D.Y., et al. (2011) RNA Interference of Caveolin-1 via Lentiviral Vector Inhibits Growth of Hypopharyngeal Squamous Cell Carcinoma Fadu Cells in Vitro and in Vivo. Asian Pacific journal of Cancer Prevention, 12, 397-401.

[22] Feng, S., Wang, Y., Wang, X., et al. (2010) Caveolin-1 Gene Silencing Promotes the Activation of PI3K/AKT Dependent on Eralpha36 and the Transformation of MCF10ACE. Science China Life Sciences, 53, 598-605.

[23] Yang, L., Qu, H., Mao, G., et al. (2013) Optimization of Subcritical Water Extraction of Polysaccharides from Grifola Frondosa Using Response Surface Methodology. Pharmacognosy Magazine, 9, 120-129. 